\title{
Three-Dimensional Organization of Chromatids by Nuclear Envelope-Associated Structures
}

\author{
GÜNTER BLOBEL \\ Laboratory of Cell Biology, The Rockefeller University Howard Hughes Medical Institute, \\ New York, New York 10065 \\ Correspondence: blobel@rockefeller.edu
}

\begin{abstract}
In evolution, the nuclear envelope (NE) arose from the prokaryotic plasma membrane. NE-associated structures, such as nuclear pore complexes (NPCs), the nuclear lamina, and nuclear envelope junctions (NEJs), have evolved to organize, among other things, chromatids within the three-dimensional space of the nucleus.NEJs represent patches of distinct integral membrane proteins of the outer and inner NE membranes, which, by interacting through conserved domains in the perinuclear space, closely align the two NE membranes. In a nuts-and-bolts configuration, the NEJs are linked to repetitive heterochromatin segments of chromatids on their nuclear side and to cytoskeletal elements on their cytoplasmic side. Cytoskeleton-generated mechanical forces are thereby effectively buffered to allow movement of nuclei in the viscous cytoplasm without disrupting the NE. Moreover, these same mechanical forces could generate distortions within the nucleus to facilitate chromatid fluctuations required for DNA repair, replication, and transcription. NPCs are the only route for bidirectional macromolecular transport between the cytoplasm and the nucleus. They also interact with euchromatin segments of chromatids. Thus far, crystallographic analyses of some nucleoporin contact sites suggest considerable plasticity. This flexibility has likely coevolved to not only buffer the mechanical forces propagated from the NEJs to the network of the more than 500 nucleoporins that make up a single NPC, but also impart fluctuations to NPC conformations for transporting large cargoes.
\end{abstract}

It took the work of a generation of cell biologists to evolve the view that the NE is more than a mere encasing for chromatids but provides signposts for their orientation within the three-dimensional space of the nucleus.

One can trace the beginning of this paradigm shift to the observation that treatment of isolated nuclei with a nonionic detergent, such as Triton X-100, did not result in the spilling of nuclear contents, such as that from a ruptured shopping bag. To the contrary, nuclei remained physically intact and retained their shape (Fig. 1) (Blobel and Potter 1966; Aaronson and Blobel 1974).

\section{ISOLATION OF THE NPC-LAMINA FRACTION}

A subsequent and more detailed analysis of the effects of Triton X-100 treatment on isolated nuclei revealed that NEembedded NPCs were not cosolubilized with the two NE membranes (95\% of the NE's lipids were) but retained their characteristic location at the distal ends of the channels leading through the peripheral heterochromatin (Blobel and Potter 1966; Aaronson and Blobel 1974). Hence, it appeared that NPCs do not require an intact NE membrane for maintaining their own structural integrity (Fig. 1).

However, because the chromatin underlying NPCs appeared to be unaffected by Triton X-100 treatment of nuclei, it remained possible that connections to chromatin are essential for maintaining the NPC's structural integrity. To test this, NE membranes were prepared by two consecutive DNase digestions of isolated rat liver nuclei (Fig. 2A). This was followed by solubilization of both membranes by the nonionic detergent Triton X-100 and salt washes to remove residual chromatin (Aaronson and Blobel 1975; Dwyer and Blobel 1976). Strikingly, NPCs were found associated with an $\sim 15$-nm-thick layer of material that was likely to represent the hitherto elusive nuclear lamina of liver cells (a nuclear lamina was thought, at that time, to be absent in liver cell nuclei) (Fig. 2B). The presumptive lamina with its attached NPCs appeared to encircle the entire nucleus but did not extend into the interior of the nucleus (Fig. 2C). The purified NPC-lamina fraction contained three major polypeptides with an estimated molecular mass of 70, 65, and 60 $\mathrm{kDa}$, in addition to other, but less abundant, polypeptides (Dwyer and Blobel 1976).

\section{IDENTIFICATION OF LAMINS A, B, AND C AS CONSTITUENTS OF THE NUCLEAR LAMINA}

Antibodies were raised to the three major polypeptides of 70,65 , and $60 \mathrm{kDa}$ of the NPC-lamina fraction. Immunofluorescence microscopy and immunoelectron microscopy localized each of these three proteins to the nuclear periphery. NPCs were spared from labeling, indicating that these three proteins were not part of the NPC. Hence, it could be concluded that the 70-, 65-, and 60$\mathrm{kDa}$ polypeptides represented constituents of the polymeric nuclear lamina and, therefore, they were termed lamins A, B, and C, respectively (Gerace et al. 1978).

During mitosis, beginning in prophase, the lamins appeared diffusely distributed in the cytoplasm, consistent with disassembly of the polymeric lamina into constituent lamins. In telophase, the lamins coalesced again around the daughter chromosome masses, indicating that, in a re- 


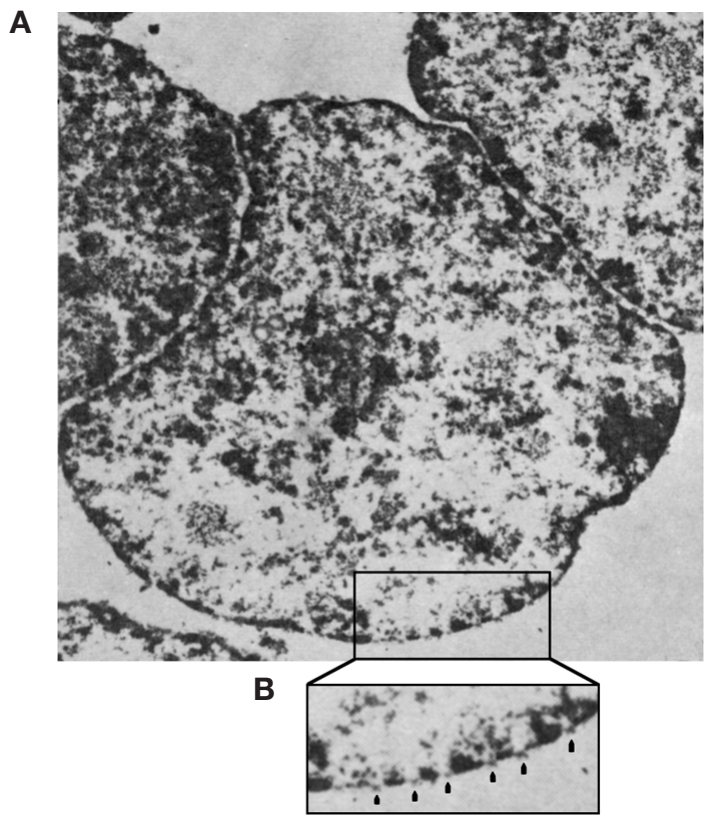

Figure 1. Isolated rat liver nuclei retain their shape after treatment with the nonionic detergent Triton X-100. (A) NPCs are barely detectable as small protrusions at the nuclear rim. (B) At higher magnifications of a boxed section of the nuclear rim, NPCs are more clearly visible and are indicated by arrows. (Modified from Blobel and Potter 1966.)

versible reaction, a lamina polymer was reformed from the depolymerized lamins in the cytoplasm. Depolymerization and repolymerization were accompanied by phosphorylation and dephosphorylation of the lamins, respectively (Gerace and Blobel 1980). Subsequently, similar kinase and phosphatase reactions also were observed to occur in the disassembly and reassembly of NPCs (see below).

Cloning and sequencing of cDNAs for the lamins showed that they are members of the family of intermediate filament proteins. Lamin $\mathrm{C}$ is coded for by an alternatively spliced mRNA transcribed from the lamin A gene (Fisher et al. 1986). Lamin A is synthesized as a larger precursor protein with a carboxy-terminal extension. Mutations in the lamin A gene are associated with a variety of diseases (laminopathies), most curiously with progeria of the Hutchinson-Gilford type and with several disorders affecting specific neurons, fat cells, and muscle cells. Subsequently, integral membrane proteins of the inner nuclear membrane were identified as lamin-interacting proteins (Worman et al. 1988; Foisner and Gerace 1993).

\section{IDENTIFICATION OF NUCLEOPORINS}

In addition to the dominant lamin polypeptides, the isolated NPC-lamina fraction contained several minor polypeptides. These were candidates for the long-soughtafter nucleoporins, a collective term coined for the constituent proteins of the NPC (Davis and Blobel 1986). To identify nucleoporins, monoclonal antibodies were raised against Triton X-100-treated rat liver nuclei. Immunofluorescence microscopy was then used as a screen to identify nucleoporin-specific antibodies. Not really knowing what to expect, we found one particular monoclonal antibody
A

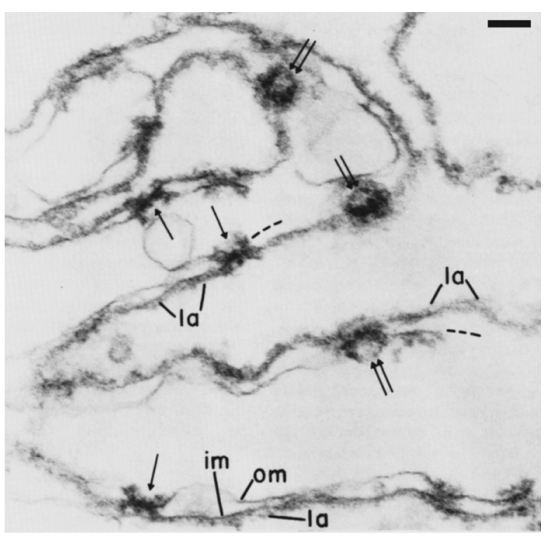

B

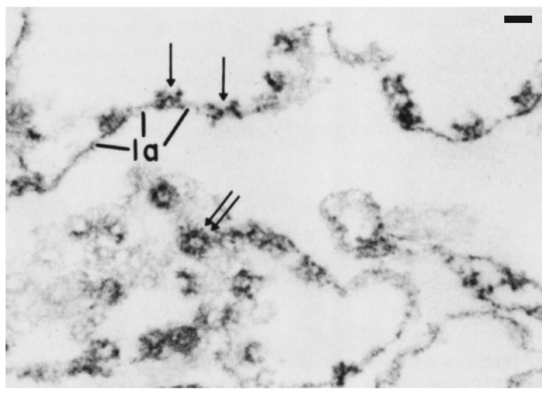

C

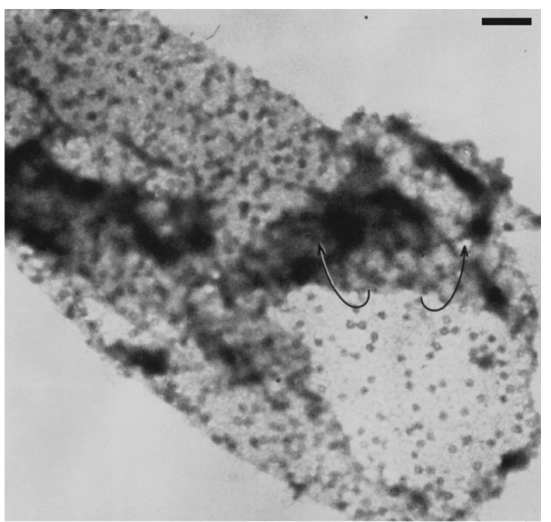

Figure 2. Isolated rat liver NEs treated with Triton X-100 yield an NPC-lamina fraction. (A) Isolated NEs with inner (im) and outer (om) NE membranes; dotted lines show pieces of om missing; lamina associated with the im is indicated, as are NPCs in lateral (single arrows) or en face (double arrows) views. Bar, 100 $\mathrm{nm}$. (B) NPC-lamina fraction; lamina (la) and NPCs (arrows) are indicated as in $A$. Bar, $100 \mathrm{~nm}$. (C) Negatively stained NPC-lamina fraction. Bar, $500 \mathrm{~nm}$; arrows indicate a flipped-over portion of the continuous NPC-lamina derived from a single NE. (Modified from Dwyer and Blobel 1976.)

$(\mathrm{mAb} 414)$ that yielded a striking punctate peripheral staining pattern, or a densely punctate surface-staining pattern when focusing on the equator or on the surface of the nucleus, respectively (Fig. 3A,B) (Davis and Blobel 1986). This punctate staining pattern had not been reported before and was consistent with the idea that each point represented an NPC in the NE. mAb 414 reacted primarily with one major protein of $62 \mathrm{kDa}$ but also cross-reacted with several other less abundant proteins. The major reactive protein of $62 \mathrm{kDa}$ was tentatively termed p62 (this protein is now known as Nup62, where Nup stands for nucleoporin and the number for the estimated molecular mass in kilodaltons, 
estimated from mobility in sodium dodecyl sulfate-polyacrylamide gel electrophoresis [SDS-PAGE] or calculated from cDNA primary structure). Immunoelectron microscopy confirmed that mAb 414 labeled NPCs, verifying that Nup62 was indeed a nucleoporin. Punctate staining in equatorial or surface views of the nucleus have since become hallmarks for NPCs.

Interestingly, Nup62 was also the first representative of a glycoprotein that was glycosylated, not by membranebound glycosyl transferases of the ER or Golgi membranes, but by a soluble cytoplasmic glycosyltransferase (Davis and Blobel 1987; Kreppel et al. 1997). Mature Nup62 contained single GlcNAc modifications linked to Ser or Thr residues. In pulse-chase experiments, Nup62 remained in a cytoplasmic fraction with a remarkable long half time of $6 \mathrm{~h}$ before it was incorporated into NPCs. This is consistent with Nup62 being synthesized continuously during the $\mathrm{G}_{1}$ phase of the cell cycle and accumulating in the cytoplasm before being incorporated into NPCs during their doubling in the following $\mathrm{S}$ phase. Incorporation of newly synthesized Nup62 appears to be accompanied by its glycosylation (Davis and Blobel 1986).

Like previously observed for the nuclear lamina, NPCs are reversibly disassembled during mitosis. Disassembly begins at prophase, with reassembly commencing at telophase (Fig. 3C-F) (Davis and Blobel 1986). Disassembly does not occur in cells with closed mitosis (e.g., yeast). Interestingly, disassembled nucleoporins (nups) or subcomplexes exert several distinct double-duty functions during various processes in mitosis.

Soon after the identification of the first nups, the entire inventory of nups was established for yeast and mam-
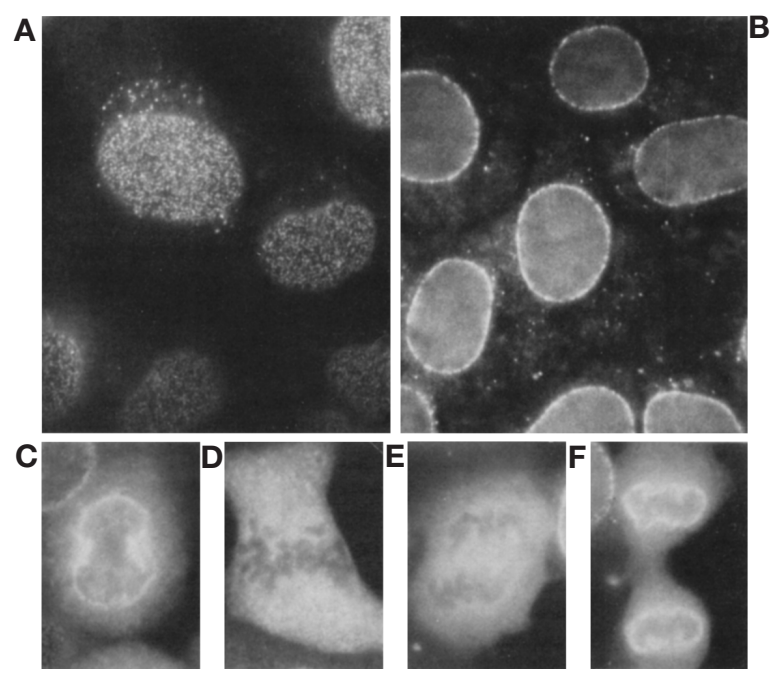

Figure 3. Identification and characterization of the first nucleoporin, Nup62, by a monoclonal antibody, mAb414. Punctate staining pattern with $\mathrm{mAb} 414$ at $(A)$ the surface or $(B)$ the equator of the nucleus. Note that punctate staining in the cytoplasm may represent cytoplasmic intermediates in NPC assembly (see text) or excess NPCs that were assembled into the endoplasmic reticulum (ER) (annulate lamellae). $(C-F)$ Concomitant with mitotic disassembly of NEs, NPCs begin to disassemble at prophase, complete their disassembly at metaphase, remain disassembled at anaphase, and reassemble at telophase. (Modified from Davis and Blobel 1986.) malian NPCs. Because of the eightfold rotational symmetry of the NPC and the twofold axis of pseudosymmetry in the plane of the membrane, the actual number of distinct nups is only about 30 . Often included in this list are the three distinct integral membrane proteins located in the pore membrane domain of the NE. These proteins are termed poms to distinguish them from nups.

\section{LOCALIZATION AND MODULAR ORGANIZATION OF NUCLEOPORINS}

The modular organization of nups and their electron microscopic localization, either to the symmetric core of the NPC or to asymmetric tentacle-like structures in the cytoplasm or nucleoplasm, provided important clues as to their function.

First, one of the modules that occurs in one-third of the nups contains phenylalanine-glycine-containing repeats, collectively termed FG repeats. These FG-containing nups are strategically localized, either asymmetrically on the nucleoplasmic or cytoplasmic side of the NPC or symmetrically, on nups lining both sides of the central 30 40-nm-wide transport channel of the NPC. The tentaclelike FG repeats are unstructured and, by serving as the universal docking sites for all transport factors, for both import into and export from the nucleus, are the business modules of nups. Nup98 was the first nup, where this module, in this case containing more than three dozen FG repeats, was discovered to interact with transport factors (Radu et al. 1995a). Crystallographic data revealed that FG residues intercalate into hydrophobic interhelical spaces of the solenoid karyopherins (Bayliss et al. 2000). Consistent with these data were atomic force microscopy analyses, in which recombinant FG repeats were immobilized on a gold chip. The immobilized and extended FG repeats were dramatically compressed and shortened from $30 \mathrm{~nm}$ to $10 \mathrm{~nm}$ following saturating binding to a karyopherin $\beta$. The compressed FG tentacles reversibly expanded again to $30 \mathrm{~nm}$ after release of karyopherin $\beta$ molecules from the FG repeats by the small GTPase Ran in its GTP-bound form (Fig. 4) (Lim et al. 2007). The demonstration of reversible compression and expansion of the unstructured FG repeat modules of nups presented a seminal advance toward understanding the mechanism of transport across the central channel of the NPC.

Second, the completion of the nup inventory revealed that one of the nups, Sec13, was identical to Sec13 of the coat protein complex II (COP II), whereas in the NPC, $\mathrm{Sec} 13$ is a member of a heptameric complex of nups. Because the other members of the heptameric complex were all predicted to be $\beta$ propellers or combinations of $\beta$ propellers and helical solenoids, and because this combination of structural folds is typical for proteins comprising the various protein complexes coating the sharp curves of membrane vesicles, the protocoatomer hypothesis was formulated (Devos et al. 2004). Because the NE, like other intracellular membranes of the exocytotic or endocytotic pathways, arose in evolution by inward folding of domains of the prokaryotic plasma membrane (Blobel 1980), these sharp bends were hypothesized to be stabilized by a group of membrane proteins, termed protocoatomers. These pro- 


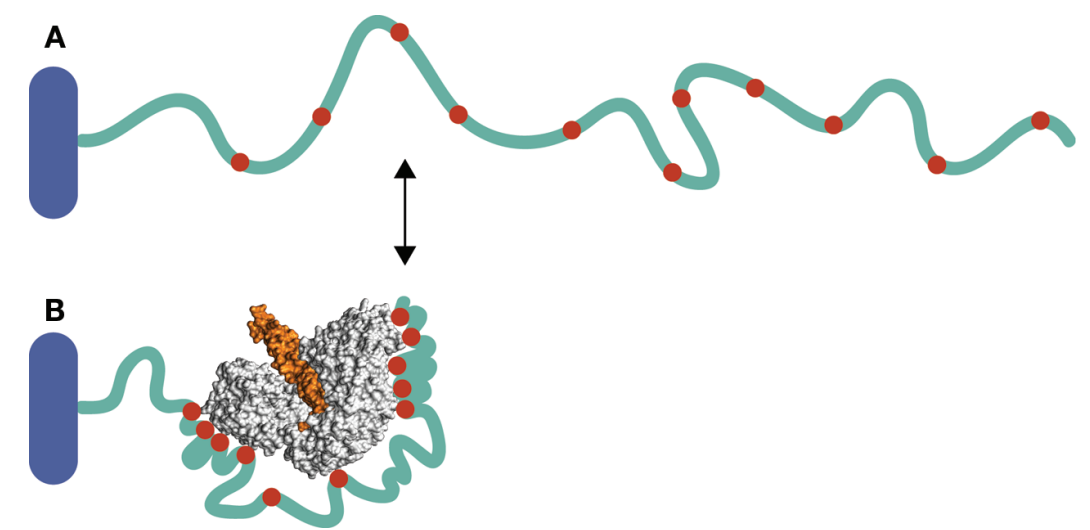

Figure 4. Reversible compression and expansion of phenylalanine-glycine (FG) repeats of a nucleoporin after association with and dissociation from karyopherin (kap) $\beta$, respectively. $(A)$ The structured part of the nup is indicated on the left by a blue oval and connects to the unstructured FG repeat region (green) with FG residues marked by red dots. $(B) \mathrm{FG}$ residues intercalate into interhelical spaces on the solenoid surface of a kap $\beta$-substrate complex (space-filling models: kap $\beta$ [gray] and substrate [orange]). After RanGTPinduced dissociation of the kap $\beta$-substrate complex, the FG regions revert again to the expanded state. (Modified from Bayliss et al. 2000; Isgro and Schulten 2005; Lim et al 2007.)

tocoatomers evolved into the many distinct coatomers that stabilize the various membrane bends in modern vesicular traffic. The evolution of the NPC probably started with the acquisition of a protocoatomer to stabilize the sharp bend between the outer and inner nuclear membrane. This was then followed by the recruitment of other proteins to evolve the unique transport and other properties of the NPC (Devos et al. 2004)

Finally, one of the nucleoporins, termed TPR (for translocated promoter region), appears to form polymers that extend from the NPC to the nuclear interior. Cablelike structures that could contain or represent polymeric TPRs have first been detected by scanning EM in amphibian eggs by Ris (1997) and were termed branching hollow cables. They form at the nuclear side of each NPC and eventually converge and anastomose as they centripetally extend from the nuclear periphery to the nuclear interior (Fig. 5A) (Ris 1997). Solubilization of TPRs from isolated NEs by heparin and a subsequent multistep fractionation yielded highly purified TPRs that, on negative staining, yielded long fibers that were $2-10 \mathrm{~nm}$ in thickness (Fig. 5B) (Shekdar 2003; K Shekdar and G Blobel, unpubl.). By immunofluorescence microscopy with antibodies to TPRs, fibrous structures extending from the nuclear side of the NPC to the nucleolus have been detected in nuclei from several organisms (Fig. 5C-E) (Zimowska et al. 1997; Fontoura et al. 2001; K Yoshida and G Blobel, unpubl.). By virtue of multiple sites of interaction of TPRs with the surface of chromatids, the TPR fibrils are proposed to form fence-like structures that delimit a relatively narrow, highly porous and irregular chromatid-free space that could represent an intranuclear system of loosely bordered and highly dynamic paths to facilitate traffic of large particles, such as ribosomal subunits between the nucleolus and the NPC. Consistent with these proposals are observations in which nucleolar proteins, such as Nopp 140 (Meier and Blobel 1992), were detected by immunoelectron microscopy in micrometer-long tracks, suggesting that they diffuse within such a network of paths connect- ing the nucleolus via NPCs to the cytoplasm (Fig. 5F).

Mammalian TPR is a protein of more than 2300 amino acid residues, with the amino-terminal two-thirds of which predicted to form a long coil with numerous bubbles, where the coiled structure is interrupted and might be a candidate site for weak interaction with a number of sites on the surface of chromatids. The carboxy-terminal third of the TPR forms an apparently nonstructured primarily acidic stretch. It is obviously urgent to learn more about TPR to refute, support, or modify these proposals.

\section{TOWARD AN ATOMIC RESOLUTION STRUCTURE OF THE NPC}

Whereas the atomic structure of large and complex organelles such as bacterial ribosomes has been solved by purifying them in large quantities and finding conditions for their crystallization, such a strategy is unlikely to succeed for NPCs. There are a number of reasons for that. To start with, although NPCs can be isolated from organisms such as yeasts, that do not contain a nuclear lamina, purified NPCs turned out to be significantly contaminated. As expected, many of the contaminants are transport substrates and transport factors that cofractionate, presumably as a result of their kinetic partitioning in the NPC (Rout and Blobel 1993). Attempts to remove these contaminants by various washing procedures led to a concomitant loss of nucleoporins. However, even if uncontaminated NPCs might one day be isolatable, the presence of the highly unstructured FG repeats would likely prevent crystallization. Moreover, electron microscope analyses have revealed that NPCs undergo large conformational changes during transport of substrates. The number of such conformational states is presently unknown.

With a deconstruction approach, one could solve atomic structures of individual nups, nup fragments, and contact sites between nups with the goal to put together larger subcomplexes of the NPC (Hsia et al. 2007; Debler et al. 2008). Electron microscope and biochemical studies sug- 


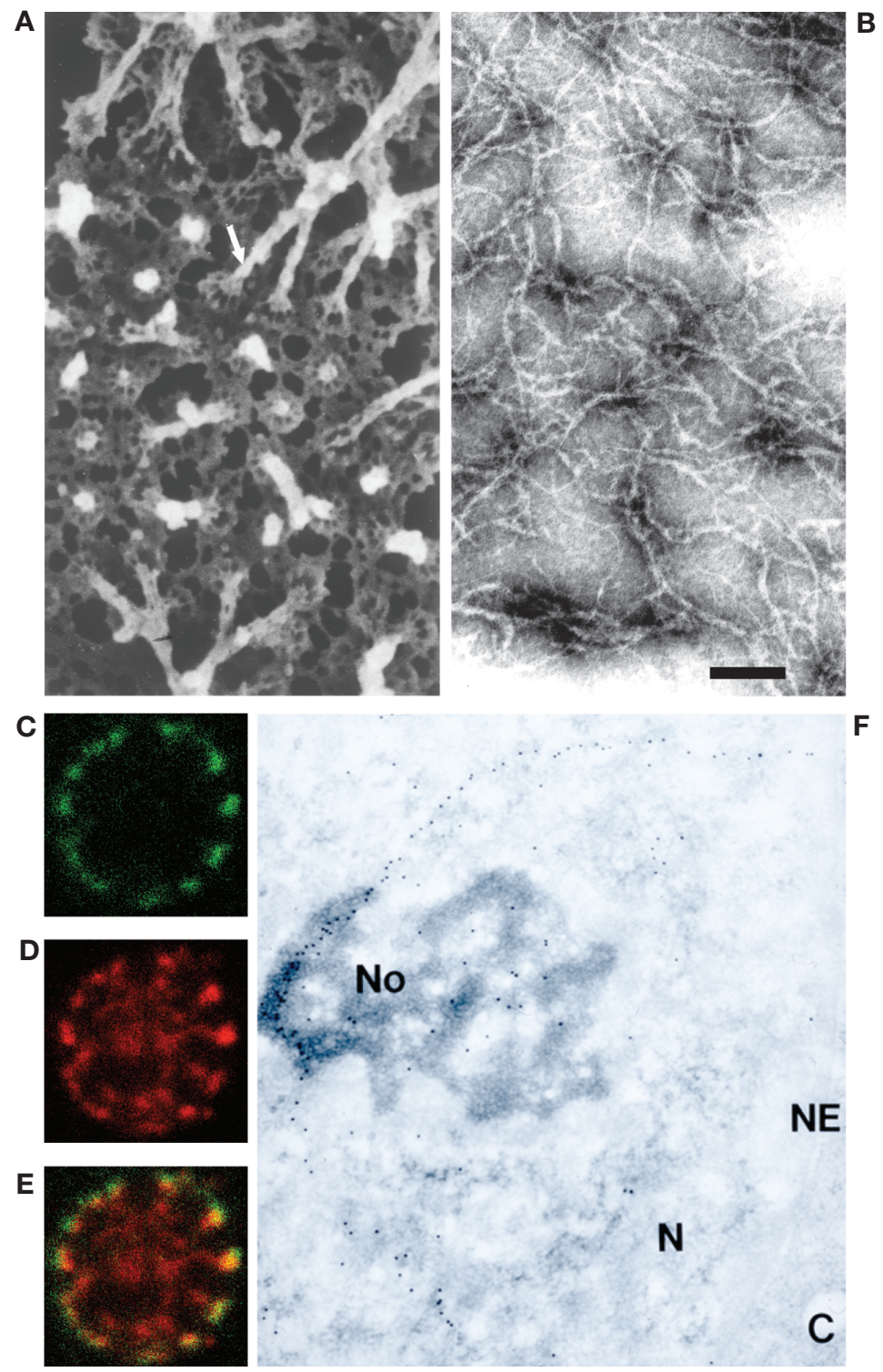

B

Figure 5. Localization and isolation of TPR, a fibrous nucleoporin that extends into the nucleoplasm. (A) View from the interior of the nucleus toward the NE of amphibian oocytes; visualization of branching hollow cables (Ris 1997) that emanate from the nuclear basket of NPCs (white arrow) toward the nuclear interior (this image is a gift from Ris [1914-2004] to the author). Ris's cables are likely to represent TPRs, at least in part (see text). (B) Biochemically purified TPR subjected to negative staining reveals its fibrous nature (Shekdar 2003; K Shekdar and G Blobel, unpubl.). Bar, $100 \mathrm{~nm}$. (C-E) Immunostaining with a monoclonal antinup antibody Mab414 (green) and an anti-TPR antibody (red) in turkey erythrocytes (K Yoshida and G Blobel, unpubl.). Note that TPR emanates from the nuclear side of NPCs and extends to the interior of the nucleus. $(F)$ Immunoelectron microscopy using cultured cells and antibodies to the shuttling nucleolar phosphoprotein Nopp140. A thin section, presumably in the plane of a virtual curvilinear cable within the nucleus, reveals numerous immunostained Nopp140 molecules aligned in a track-like fashion between the nucleolus (No) and the cytoplasm (C); (NE) nuclear envelope, (N) nucleus. The track-like configurations might represent transport in a TPR-delimited, chromatid-free zone extending from the nucleolus to the NPC. (Modified from Meier and Blobel 1992.) gest that the NPC consists of a symmetrical core with an eightfold axis of symmetry (some NPCs contain sevenfold or ninefold axes, indicating a degree of structural degeneracy of the NPC). The symmetric core nups do not contain FG repeats, except for those that line the channel of the NPC. In addition to their FG repeats, the channel nups contain helical modules that appear to anchor these nups to the NPC. The latter modules can form complexes either by themselves or with ordered domains of neighboring nups (I Melcák and G Blobel, unpubl.). A similar structural modularity also exists for asymmetrically oriented nups that, in addition to the unstructured FG repeats, contain structured modules that anchor these proteins to the NPC.

Assuming that $\sim 30$ nups and poms contact, on average, three other nups or poms, an estimated 50 distinct contact surfaces may exist in the NPC. So far, more than a dozen specific contact sites comprising up to three nups (Nagy et al. 2009) have been defined at atomic resolution. Several conclusions can be drawn from these data.
Some nups form conjugating pairs. The Sec13/Nup145C pair (Hsia et al. 2007) and the Seh1/Nup85 pair (Debler et al. 2008) represent situations where a six-bladed propeller of either Sec13 or Seh1 is invaded by a $\beta$-stranded region of Nup145C or Nup85, respectively (Fig. 6A). In each case, the invasion motif contributes a seventh blade to the propeller. Conjugation can be promiscuous, because the sixbladed propeller of Sec13 can also be invaded by Sec31 to provide a seventh blade for Sec13 and, in this case, to form a dimeric unit of the COP II complex (Fath et al. 2007). The mechanism that directs Sec13 into the NPC or COP II complex assembly is not presently known. Besides conjugation via their invasion domains, there is an additional contact site between the solenoid portion of Nup145C or Nup85 and their cognate seven-bladed $\beta$ propellers (Hsia et al 2007; Debler et al. 2008).

Moreover, each of the conjugated pairs was found to form tetramers and octamers. Tetramers are formed via association of two Sec13/Nup145C conjugates via their 
A

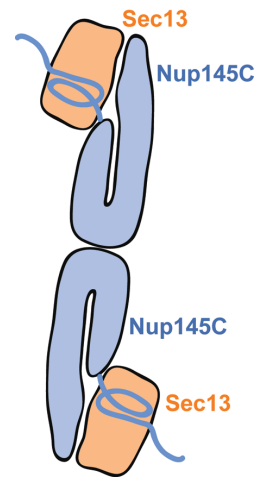

B

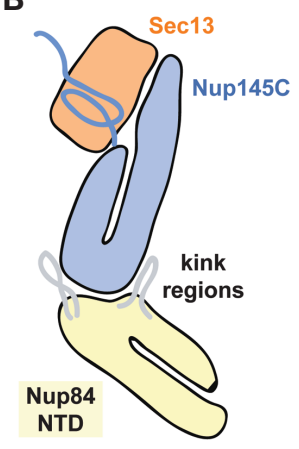

Figure 6. Two crystal forms reveal alternative contacts between nucleoporins. (A) The Sec13/Nup145C nucleoporin conjugate can form either a tetramer with another Sec13/Nup145C conjugate or $(B)$ a heterotrimer by interacting with the amino-terminal domain (NTD) of Nup84. (Modified from Hsia et al. 2007; Nagy et al 2009.)

Nup145C surfaces or of two Seh1/Nup85 conjugates via their Nup85 surfaces (Fig. 6A) (Hsia et al. 2007; Debler et al. 2008). For the Seh1/Nup85 tetramer, distinct crystal structures suggested the existence of several conformers, where the angle at the tetrameric interface between two conjugates is tilted by $30^{\circ}$ or where the long axis of the tetrameric interface is rotated by $80^{\circ}$ between the two conjugates (Debler et al. 2008). These snapshots are likely to reflect different conformers in vivo. Octamers are formed from tetramers using their terminal Sec13 or Seh1 components, respectively. Here again, the $\beta$ propellers form additional contact sites with the corresponding solenoids of Nup145C or Nup85. A Sec13/Sec31 octamer appears to be the basic unit of COP II.

The design principle of promiscuity of contact sites between nups became evident again when the trimeric Sec13/Nup145C/Nup84 complex was analyzed. Instead of interacting with a Nup145C molecule of another Sec13/Nup145C conjugate to form a tetramer (Hsia et al. 2007), the surface of the Nup145C molecule of the Sec13/Nup145C conjugate interacts with Nup84, yielding a Sec13/145C/Nup84 trimer (Fig. 6A vs. 6B) (Nagy et al. 2009). It is possible that both conformers occur in the NPC. Alternatively, they could represent distinct intermediates in the assembly pathways for NPCs.

A most dramatic example of flexibility was gleaned from a helical region of the central channel nucleoporin Nup58. Apposing amphipathic helices containing ribbons of large hydrophilic residues were displaced in the two crystal forms by two helical turns (i.e., $11 \AA$ ) (Fig. 7). These two snapshots suggested that the displaced helices might represent various degrees of sliding with respect to each other. With eight such helical modules lining the central channel of the NPC, its diameter could potentially be dilated by $3 \mathrm{~nm}$ (Melcák et al. 2007). It is likely that more of these examples will be found, particularly for the nups lining the highly flexible central channel of the NPC. Large fluctuations in the central channel nucleoporins are likely to be buffered by concomitant dynamic changes in contact sites between the neighboring nups and eventually reverberate throughout

the dynamic network of contact sites between the more than 500 nups that constitute an NPC. This highly dynamic network of nup contact sites may have arisen in evolution to not only accommodate the large size range of transport substrates, but also, hypothetically, withstand the mechanical forces exerted by nuclear envelope junctions (NEJs) (see below). Moreover, the dynamic network of contact sites may also facilitate coordinated access to the numerous enzymes that are expected to perform the many posttranslational modifications of nups and poms that occur concomitantly with assembly and disassembly of the NPC.

Another striking example for the plasticity of contact sites between nups and poms in the assembled NPC became apparent when the cellular pathway for an integral membrane protein of the inner nuclear membrane was investigated. Like all other integral membrane proteins, which are residents of the inner nuclear membrane or the membranes of exocytotic and endocytotic pathways, the asymmetric integration into the membrane occurs exclusively in the rough ER (or the equivalent outer nuclear membrane). Their asymmetric integration requires the rough ER-associated machineries, such as signal recognition particle (SRP), SRP receptor, and, most importantly, the protein-conducting channel. Strikingly, an integral membrane protein that resides in the inner nuclear membrane was found to contain a nuclear localization sequence (NLS) on its nucleoplasmic domain, which mediates karyopherin- and RanGTP-mediated transport of the nucleoplasmic domain through the transport channel of the NPC (see below). Concomitantly with transporting the soluble nucleoplasmic domain through the central transport channel of the NPC, the transmembrane segment(s) and their extensions have to literally cut across the nucleocytoplasmic axis of the assembled nups and poms of the NPC to reach their destination at the inner nuclear membrane (King et al. 2006). One possibility is that the interactions between the eight vertical slices of the NPC in the nucleocytoplasmic axis are weak and highly dynamic so as to easily accommodate such a traversal without significantly disrupting the NPC.

\section{GENE GATING}

Early electron microscopic images already showed that the less compacted portions of chromatids (collectively called euchromatin) are located subjacent to NPCs, suggesting specific connections between NPCs and euchromatin. In contrast, the compacted portions of chromatids (collectively termed heterochromatin) are located mostly in the nuclear periphery, between NPCs. A biochemical association between nucleoporins on the nuclear side of NPCs and euchromatin would provide numerous signposts for organizing the euchromatin portions of the chromatids within the three-dimensional confines of the nucleus (Blobel 1985). Such arrangements would increase accessibility of euchromatin to cytoplasmic factors and facilitate transport of transcription products into the cytoplasm (for an organizing role of TPR filaments, see above). It was recently reported that, on activation, an inducible gene in yeast localizes from the interior of the nucleus to NPCs. Specific DNA sequence elements were identified interacting with a 

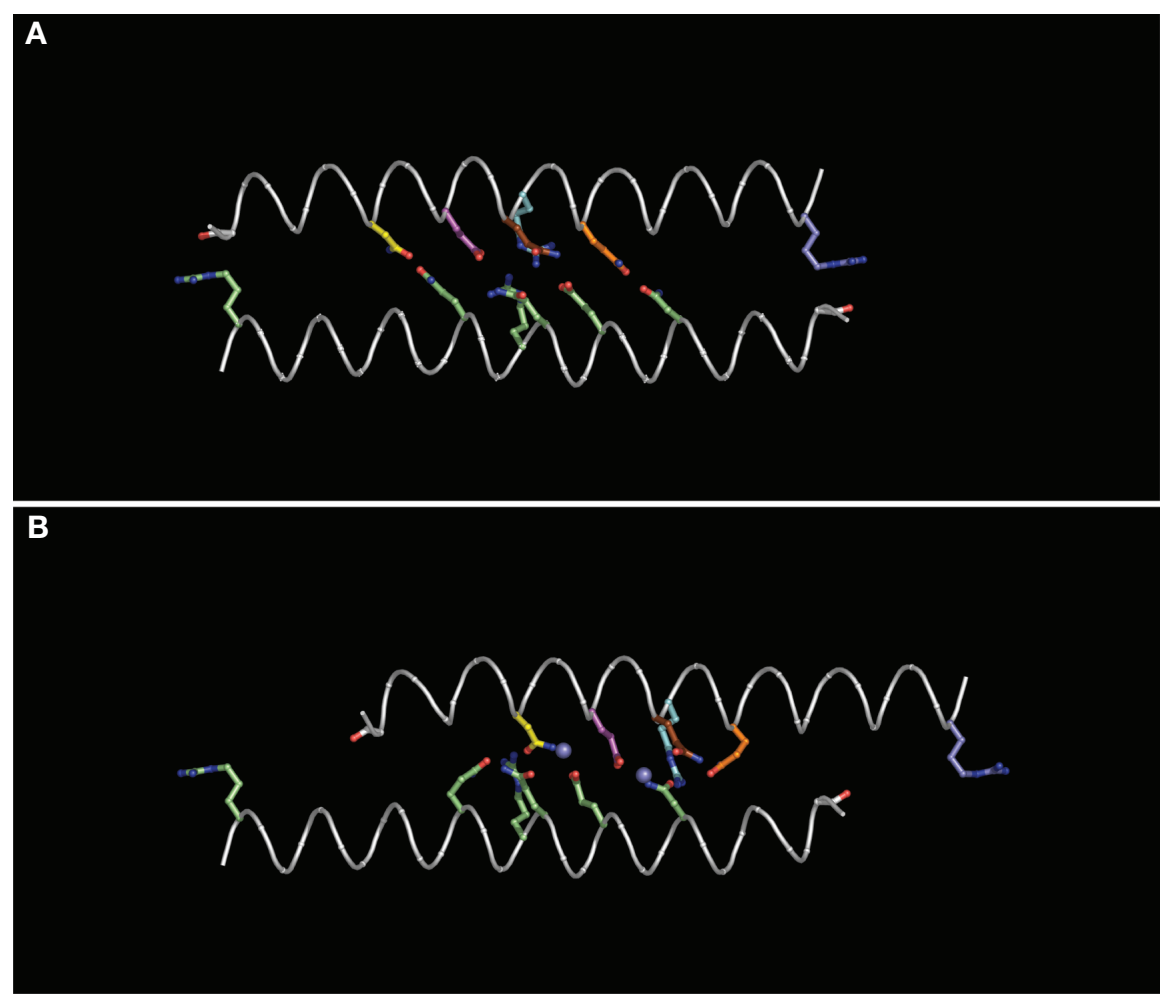

Figure 7. Dynamic interaction between an $\alpha$ helical portion of a central channel nucleoporin, Nup58. (Top and bottom) Two crystal forms of a helical portion of the central channel nucleoporin Nup58 revealed alternative contacts between apposed Nup58 amphipathic helices. The apposed hydrophilic ribbons of the amphipathic helices are composed of large hydrophilic residues. Note the displacement of the two apposing helices by two helical turns (11 A). (Modified from Melcák et al. 2007.)

specific nup on the nucleoplasmic side of the NPC (Ahmed et al. 2010). Moreover, messenger RNA (mRNA) export, histone deubiquitination, and transcription have been linked to gene gating at the NPC (Cabal et al. 2006; Köhler et al. 2008; Jani et al. 2009; Ellisdon et al. 2010)

\section{TRANSPORT FACTORS}

The pioneering development of an in vitro system for transport into the nucleus led to the identification of cytoplasmic transport factors. This in vitro system used low concentrations of digitonin to solubilize much of the cholesterol-rich plasma membrane of cultured cells, leaving other, less cholesterol-rich intracellular membranes, particularly the NE membrane, intact. Concomitant with the solubilization of parts of the plasma membrane, much of the cellular cytosol leaks out and could therefore be easily washed away. In a semiquantitative assay, import of a fluorescently labeled import substrate into the nuclei of these digitonin-permeabilized and washed cells could be monitored and shown to require addition of cytosol and energy (Adam et al. 1990). A further technical improvement was achieved by more rigorously quantifying the import reaction. This was done by digitizing the fluorescent signal and averaging it from a randomly designated area in each nucleus in a field of nuclei. These procedures yielded quantitative data that greatly facilitated the monitoring of biochemical purification of various transport factors from the cytosol (Moore and Blobel 1992). The first and most abundant transport factor that was purified from the cytosol using this system turned out to be Ran, a small Ras-related nuclear GTPase of hitherto unknown function (Moore and Blobel 1993). Following identification of Ran as an import factor, the kap $\beta$ and kap $\alpha$ families of transport factors were purified and characterized independently by several laboratories (see, e.g., Moroianu et al. 1995; Radu et al. 1995b). Kap $\alpha$ acts as an adapter that binds to both an NLS of an import substrate and Kap $\beta-1$. Most of the other, more than a dozen, $\beta$ kaps bind either directly to NLS (import kaps) or, in the case for export kaps, to the nuclear export sequence of proteins. Some $\beta$ kaps also bind to small RNAs and function in their export (Cook et al. 2009). Curiously, one of the $\beta$ kaps, Kap142p in yeast, can function either as an import or an export kap (Yoshida and Blobel 2001).

In both interphase and mitotic cells, RanGTP functions principally as a $\beta$ kap-binding protein, affecting the binding affinity of various import and export $\beta$ kaps to their cognate substrates.

In interphase cells, a gradient of RanGTP/RanGDP across the NE is maintained (see below). In the nucleus, the higher concentration of RanGTP leads to the formation of a ternary complex with an export $\beta$ kap and its cognate export substrate. The highly abundant RanGTP in the nucleus also forms a binary complex with an import $\beta$ kap, thereby dissociating the import substrate from the cognate import $\beta$ kap (Rexach and Blobel 1995). NPCs have an im- 
portant role in the kinetic partitioning of RanGTP and RanGDP by providing numerous direct binding sites (e.g., Nup358 on the cytoplasmic side of the NPC or Nup153 on the nucleoplasmic side of the NPC). Moreover, various Ran regulatory proteins, such as Ran GTPase activating protein (RanGAP) and RanGDP/GTP exchange factor, are highly concentrated at the NPC. Virtually all of the cellular RanGAP is partitioned at Nup358 at the cytoplasmic side of the NPC (Matunis et al. 1996; Mahajan et al. 1997). Targeting of RanGAP to Nup358 is accomplished by sumoylation of RanGAP via the Sumo E3 activity module of Nup358 (Pichler et al. 2004). This is just one example of nup modules kinetically partitioning transport catalysts. Another is the localization of the DEAD-box helicase Ddx19 at Nup214 on the cytoplasmic side of the NPC (Napetschnig et al. 2009) (see below).

In mitotic cells, the interphase RanGTP/RanGDP gradient between the nucleus and the cytoplasm is eliminated following disassembly of the NE during prophase. As a result, RanGTP, RanGDP, and the corresponding regulatory factors (see below) are strikingly repartitioned in the mitotic cell to facilitate cooperative assembly of mitosisspecific complexes (e.g., kinetochore, mitotic spindle, etc.) from numerous substrates, using $\beta$ kaps and RanGTP as delivery vehicles.

Members of the kap $\alpha$ and kap $\beta$ families are built of helical repeats stacked on top of one another to form a highly flexible superhelix (Conti et al. 1998; Chook and Blobel 1999). Atomic structure analysis of a Kap $\beta-2$ complex with its cognate NLS resulted in the elucidation of an NLS consensus sequence that is specific for Kap $\beta-2$. Bioinformatic analyses then allowed the detection of this consensus sequence in numerous proteins, all of which are candidates for using Kap $\beta$-2 for their import into the nucleus (Lee et al. 2006). Future analyses of other kap $\beta$ substrate complexes will determine the sequence elements for cognate $\beta$ kaps for the numerous proteins with a nuclear lifestyle.

In addition to kaps, there are other transport factors, e.g., NTF2 (import of RanGDP into the nucleus), snurportin (a kap $\alpha$-like adapter for snRNP import), or the various transport factors that cooperate in the export of mRNPs or ribosomal subunits. All of these transport factors relay their cognate cargo across the NPC via the FG repeat modules of nups.

A particularly interesting case is mRNP export. mRNAs are coated by a family of mRNA-binding proteins (next to histones and Ran, the most abundant proteins in the nucleus). Some of these mRNA-binding proteins recognize specific regions of $\mathrm{mRNA}$ such as the $5^{\prime} \mathrm{CAP}$ or the poly(A) region. Others recognize specific secondary structures of mRNA. However, the bulk of mRNA-binding proteins function to neutralize the negative charge of the phosphate backbone of the RNA with only weak specificity for bases. These proteins coat the mRNA, on average, at one protein molecule per every 15 nucleotides. Hence, depending on the length of mRNA, a given mRNP may contain hundreds of such mRNA-binding proteins. After presumably diffusing in the chromatin-free zones (delimited by TPR; see above) to the nucleoplasmic side of the NPC, the $5^{\prime}$ end of mRNA (bound to the CAP-binding protein) is thought to enter the NPC (Mehlin et al. 1992), with the remainder of the mRNP transported by a Brownian ratchet mechanism through the central channel of the NPC (Simon et al. 1992; Stewart 2007). The helicase Ddx19 is concentrated at Nup214. In an ATPase cycle that involves reversible dissociation of the helicase from Nup214, the helicase strips off one mRNA-binding protein for each ATPase cycle (Napetschnig et al. 2009; von Moeller et al. 2009). It is then thought that a ribosome, occupying 30 nucleotides (i.e., the equivalent of two mRNAbinding proteins) binds to the temporarily protein-free portion of the emerging $5^{\prime}$ end of the mRNA and begins translation on the cytoplasmic side of the NPC. As more mRNA-binding proteins are stripped off, more ribosomes bind, thereby completing the Brownian ratchet until all of the mRNA has traversed the central channel of the NPC. In this transport process, most, if not all, nuclear mRNA-binding proteins are replaced by cytoplasmic equivalents (e.g., CAP-binding protein, poly[A]-binding protein), whereas the nuclear mRNA-binding proteins are reimported into the nucleus for another cycle of cotranscriptional mRNP assembly and diffusion of the mRNP to the NPC.

\section{NUCLEAR ENVELOPE JUNCTIONS}

Through evolutionarily conserved perinuclear domains termed SUN domains for integral proteins of the inner nuclear membrane and KASH domains for integral membrane proteins of the outer nuclear membrane, and through the proposed formation of patches comprised of perhaps hundreds of these and other associated integral membrane proteins, the two membranes of the NE are closely aligned, forming NEJs. The nucleoplasmic domains of the inner membrane proteins interact through a relay of proteins (largely undefined and uncharacterized) with repetitive regions of chromatids (such as centromeres or telomeres). The cytoplasmic domains of the outer membrane proteins likewise interact, directly or indirectly, with microtubules, actin, and intermediate filaments. The stabilizing linkages to heterochromatin and the cytoskeleton of NEJs on both sides of the NE were proposed to yield a nuts-and-bolts configuration that evolved to withstand the mechanical forces exerted by the cytoskeleton on the integrity of the NE (King et al. 2008). In fact, genetic deletion in Schizosaccharomyces pombe of one inner membrane protein from this patch of NEJ proteins led to an impairment of NE integrity (King et al. 2008). The mechanical forces exerted actively (actin- and microtubule-based motors) or passively (intermediate filaments) allow movement and positioning of the large nucleus within the viscous cytoplasm without disrupting the integrity of the NE and its underlying structures. They were also suggested to facilitate the large-range chromatid fluctuations that must occur within the crowded environment of the nucleus to align chromatids for DNA repair, DNA replication, or transcription (King et al. 2008). In addition, it is proposed here that these mechanical forces also impacted on the evolution of NPCs, yielding a network of nucleoporins that had to be sufficiently flexible to withstand disruption of the NPC. The evolution of a flexible dynamic network of nups also served to accommodate the 
transport of large substrates. Hence, the NPC evolved as a wobbly transport organelle with modules of reversibly compacted FG repeats, sliding helices, and multiple alternative contacts between nucleoporins and other, yet to be discovered, flexible structures. Collectively, this flexibility allows for the large conformational changes that make it the most versatile and dynamic transport organelle of the cell.

\section{CONCLUSIONS}

The two major NE-associated structures, NPCs and the lamina, were discovered using electron microscopy in the 1950s. Their biochemical characterization was not accomplished until more than a dozen years later, leading to the foundation of large fields of research. A few years ago, connections between the inner and outer nuclear membranes, here termed NEJs, have been discovered by genetic and cell biological approaches.

The NEJs are formed by a family of integral membrane proteins of the inner and outer nuclear membranes that interact through conserved sequences in their perinuclear domains and form large patches in the NE, thereby bringing the two membranes into close apposition. On the nuclear side, NEJs are indirectly linked to heterochromatin portions of chromatids (such as centromeres and telomeres) and on the cytoplasmic side to cytoskeletal elements. These nuts-and-bolts-like configurations on either side of the NEJs stabilize them to withstand the potentially disruptive mechanical forces during motoring of the very large nucleus through the viscous cytoplasm to achieve proper positioning of the nucleus. Moreover, the mechanical forces transmitted via NEJs are also likely to facilitate the large intranuclear fluctuations of chromatids required for DNA repair, DNA replication, and transcription. Finally, it was proposed here that the mechanical forces emanating from the cytoskeleton via the NEJs also impact the large NPC that is composed of more than 500 proteins. Beginning atomic structure analyses of some contact sites between the more than 30 nucleoporins and pore membrane proteins that comprise the NPC have revealed new concepts in its structural design that begin to explain the highly dynamic and wobbly character of this largest of the cellular transport organelles. These design features include sliding helices, alternating contact sites between nups, and reversible compression of natively unfolded FG modules of nups and are thought not only to buffer the mechanical wear and tear emanating from NEJs and associated structures but also to have favored the evolution of a transport organelle that can handle a large size range of transport substrates. Much remains to be done in this burgeoning field. Multidisciplinary approaches will be required to yield a better understanding of the function of the NEassociated structures and their myriad of dynamic contacts with chromatids and cytoplasmic components.

The present deconstruction approach toward solving the atomic structure of the NPC has already revealed some of the principles governing the dynamics of the nucleoporin interactome. Subsequent reconstruction of higher-order structures and their analysis by cryoelectron microscopy (and fitting in the already elucidated atomic structures) will likely provide critical information about the repertoire of atomic interactions among the various nups and poms. To decipher the interactions that are relevant for preassembly, co-assembly, or postassembly reactions of the NPC as well as for the assembly of organelles of the mitotic apparatus will, in addition to the currently practiced deconstruction and reconstruction strategies, require biophysical, genetic, and cell biological approaches.

\section{ACKNOWLEDGMENTS}

This short review of the time line of conceptual advances in the field of structure and function of the NE and its associated structures is obviously subjective. A vast number of relevant references has been omitted due to space limitations. The reader is referred to numerous specialized reviews. I thank past and present members of our laboratory for numerous stimulating and enlightening discussions during the past 40 years. Elias Coutavas, Ivo Melcak, and Vivien Nagy helped in the preparation of the figures, and Elias Coutavas, Erik Debler, and Andre Hoelz helped in corrections of this manuscript.

\section{REFERENCES}

Aaronson RP, Blobel G. 1974. On the attachment of the nuclear pore complex. J Cell Biol 62: 746-754.

Aaronson RP, Blobel G. 1975. Isolation of nuclear pore complexes in association with a lamina. Proc Natl Acad Sci 72: 1007-1011.

Adam SA, Marr RS, Gerace L. 1990. Nuclear protein import in permeabilized mammalian cells requires soluble cytoplasmic factors. J Cell Biol 111: 807-816.

Ahmed S, Brickner DG, Light WH, Cajigas I, McDonough M, Froyshteter AB, Volpe T, Brickner JH. 2010. DNA zip codes control an ancient mechanism for gene targeting to the nuclear periphery. Nat Cell Biol 12: 111-118.

Bayliss R, Littlewood T, Stewart M. 2000. Structural basis for the interaction between FXFG nucleoporin repeats and importin- $\beta$ in nuclear trafficking. Cell 102: 99-108.

Blobel G. 1980. Intracellular protein topogenesis. Proc Natl Acad Sci 77: 1496-1500.

Blobel G. 1985. Gene gating: A hypothesis. Proc Natl Acad Sci 82: $8527-8529$.

Blobel G, Potter VR. 1966. Nuclei from rat liver: Isolation method that combines purity with high yield. Science 154: 1662-1665.

Cabal GG, Genovesio A, Rodriguez-Navarro S, Zimmer C, Gadal O, Lesne A, Buc H, Feuerbach-Fournier F, Olivo-Marin JC, Hurt $\mathrm{EC}$, et al. 2006. SAGA interacting factors confine sub-diffusion of transcribed genes to the nuclear envelope. Nature 441: 770 773.

Chook YM, Blobel G. 1999. Structure of the nuclear transport complex karyopherin-32-Ran x GppNHp. Nature 399: 230-237.

Conti E, Uy M, Leighton L, Blobel G, Kuriyan J. 1998. Crystallographic analysis of the recognition of a nuclear localization signal by the nuclear import factor karyopherin $\alpha$. Cell 94: 193-204.

Cook AG, Fukuhara N, Jinek M, Conti E. 2009. Structures of the tRNA export factor in the nuclear and cytosolic states. Nature 461: $60-65$

Davis LI, Blobel G. 1986. Identification and characterization of a nuclear pore complex protein. Cell 45: 699-709.

Davis LI, Blobel G. 1987. Nuclear pore complex contains a family of glycoproteins that includes p62: Glycosylation through a previously unidentified cellular pathway. Proc Natl Acad Sci 84: 7552-7556.

Debler EW, Ma Y, Seo HS, Hsia KC, Noriega TR, Blobel G, Hoelz A. 2008. A fence-like coat for the nuclear pore membrane. $\mathrm{Mol}$ Cell 32: 815-826. 
Devos D, Dokudovskaya S, Alber F, Williams R, Chait BT, Sali A, Rout MP. 2004. Components of coated vesicles and nuclear pore complexes share a common molecular architecture. PLoS Biol 2: 2085-2093.

Dwyer N, Blobel G. 1976. A modified procedure for the isolation of a pore complex-lamina fraction from rat liver nuclei. $J$ Cell Biol 70: 581-591.

Ellisdon AM, Jani D, Köhler A, Hurt E, Stewart M. 2010. Structural basis for the interaction between yeast Spt-Ada-Gen5 acetyltransferase (SAGA) complex components Sgf11 and Sus1. J Biol Chem 285: 3850-3856.

Fath S, Mancias JD, Bi X, Goldberg J. 2007. Structure and organization of coat proteins in the COPII cage. Cell 29: 1325-1336.

Fisher DZ, Chaudhary N, Blobel G. 1986. cDNA sequencing of nuclear lamins $\mathrm{A}$ and $\mathrm{C}$ reveals primary and secondary structural homology to intermediate filament proteins. Proc Natl Acad Sci 83: 6450-6454.

Foisner R, Gerace L. 1993. Integral membrane proteins of the nuclear envelope interact with lamins and chromosomes, and binding is modulated by mitotic phosphorylation. Cell 73: 1267-1279.

Fontoura BM, Dales S, Blobel G, Zhong H. 2001. The nucleoporin Nup98 associates with the intranuclear filamentous protein network of TPR. Proc Natl Acad Sci 98: 3208-3213.

Gerace L, Blobel G. 1980. The nuclear envelope lamina is reversibly depolymerized during mitosis. Cell 19: 277-287.

Gerace L, Blum A, Blobel G. 1978. Immunocytochemical localization of the major polypeptides of the nuclear pore complexlamina fraction. Interphase and mitotic distribution. J Cell Biol 79: $546-566$.

Hsia KC, Stavropoulos P, Blobel G, Hoelz A. 2007. Architecture of a coat for the nuclear pore membrane. Cell 131: 1313-1326.

Isgro TA, Schulten K. 2005. Binding dynamics of isolated nucleoporin repeat regions to importin- $\beta$. Structure 12: 1869-1879.

Jani D, Lutz S, Marshall NJ, Fischer T, Köhler A, Ellisdon AM, Hurt E, Stewart M. 2009. Sus1, Cdc31, and the Sac3 CID region form a conserved interaction platform that promotes nuclear pore association and mRNA export. Mol Cell 33: 727-737.

King MC, Lusk CP, Blobel G. 2006. Karyopherin-mediated import of integral inner nuclear membrane proteins. Nature 442: 1003 1007.

King MC, Drivas TG, Blobel G. 2008. A network of nuclear envelope membrane proteins linking centromeres to microtubules. Cell 134: 427-438.

Köhler A, Schneider M, Cabal GG, Nehrbass U, Hurt E. 2008. Yeast Ataxin-7 links histone deubiquitination with gene gating and mRNA export. Nat Cell Biol 6: 707-715.

Kreppel LK, Blomberg MA, Hart GW. 1997. Dynamic glycosylation of nuclear and cytosolic proteins. Cloning and characterization of a unique $O$-GlcNAc transferase with multiple tetratricopeptide repeats. J Biol Chem 272: 9308-9315.

Lee BJ, Cansizoglu AE, Süel KE, Louis TH, Zhang Z, Chook YM. 2006. Rules for nuclear localization sequence recognition by karyopherin $\beta 2$. Cell 126: 543-558.

Lim RY, Fahrenkrog B, Köser J, Schwarz-Herion K, Deng J, Aebi U. 2007. Nanomechanical basis of selective gating by the nuclear pore complex. Science 318: 640-643.

Mahajan R, Delphin C, Guan T, Gerace L, Melchior F. 1997. A small ubiquitin-related polypeptide involved in targeting RanGAP1 to nuclear pore complex protein RanBP2. Cell 88: 97-107.

Matunis MJ, Coutavas E, Blobel G. 1996. A novel ubiquitin-like modification modulates the partitioning of the Ran-GTPase-activating protein RanGAP1 between the cytosol and the nuclear pore complex. J Cell Biol 135: 1457-1470.

Mehlin H, Daneholt B, Skoglund U. 1992. Translocation of a spe- cific premessenger ribonucleoprotein particle through the nuclear pore studied with electron microscope tomography. Cell 69: 605-613.

Meier UT, Blobel G. 1992. Nopp140 shuttles on tracks between nucleolus and cytoplasm. Cell 70: 127-138.

Melcák I, Hoelz A, Blobel G. 2007. Structure of Nup58/45 suggests flexible nuclear pore diameter by intermolecular sliding. Science 315: 1729-1732.

Moore MS, Blobel G. 1992. The two steps of nuclear import, targeting to the nuclear envelope and translocation through the nuclear pore, require different cytosolic factors. Cell 69: 939-950.

Moore MS, Blobel G. 1993. The GTP-binding protein Ran/TC4 is required for protein import into the nucleus. Nature 365: 661-663.

Moroianu J, Blobel G, Radu A.1995. Previously identified protein of uncertain function is karyopherin $\alpha$ and together with karyopherin $\beta$ docks import substrate at nuclear pore complexes. Proc Natl Acad Sci 92: 2008-2011.

Nagy V, Hsia KC, Debler EW, Kampmann M, Davenport AM, Blobel G, Hoelz A. 2009. Structure of a trimeric nucleoporin complex reveals alternate oligomerization states. Proc Natl Acad Sci 106: $17693-17698$.

Napetschnig J, Kassube SA, Debler EW, Wong RW, Blobel G, Hoelz A. 2009. Structural and functional analysis of the interaction between the nucleoporin Nup214 and the DEAD-box helicase Ddx19. Proc Natl Acad Sci 106: 3089-3094.

Pichler A, Knipscheer P, Saitoh H, Sixma TK, Melchior F. 2004. The RanBP2 SUMO E3 ligase is neither HECT- nor RINGtype. Nat Struct Mol Biol 10: 984-991.

Radu A, Blobel G, Moore MS. 1995a. Identification of a protein complex that is required for nuclear protein import and mediates docking of import substrate to distinct nucleoporins. Proc Natl Acad Sci 92: 1769-1773.

Radu A, Moore MS, Blobel G. 1995b. The peptide repeat domain of nucleoporin Nup98 functions as a docking site in transport across the nuclear pore complex. Cell 81: 215-222.

Rexach M, Blobel G. 1995. Protein import into nuclei: Association and dissociation reactions involving transport substrate, transport factors, and nucleoporins. Cell 83: 683-692.

Ris H. 1997. High-resolution field-emission scanning electron microscopy of nuclear pore complex. Scanning 19: 368-375.

Rout MP, Blobel G. 1993. Isolation of the yeast nuclear pore complex. J Cell Biol 123: 771-783.

Shekdar K. 2003. "Intranuclear filamentous protein network: Purified Tpr protein forms fibers in vitro and endogenous Tpr is localized to structures consistent with intranuclear filamentous protein networks in vivo. " $\mathrm{PhD}$ thesis, The Rockefeller University, New York.

Simon SM, Peskin CS, Oster GF. 1992. What drives the translocation of proteins? Proc Natl Acad Sci 89: 3770-3774.

Stewart M. 2007. Ratcheting mRNA out of the nucleus. Mol Cell 25: $327-330$.

von Moeller H, Basquin C, Conti E. 2009. The mRNA export protein DBP5 binds RNA and the cytoplasmic nucleoporin NUP214 in a mutually exclusive manner. Nat Struct Mol Biol 16: $247-254$.

Worman HJ, Yuan J, Blobel G, Georgatos SD. 1988. A lamin B receptor in the nuclear envelope. Proc Natl Acad Sci 85: 85318534.

Yoshida K, Blobel G. 2001. The karyopherin Kap142p/Msn5p mediates nuclear import and nuclear export of different cargo proteins. J Cell Biol 152: 729-740.

Zimowska G, Aris JP, Paddy MR. 1997. A Drosophila Tpr protein homolog is localized both in the extrachromosomal channel network and to nuclear pore complexes. J Cell Sci 110: 927-944. 


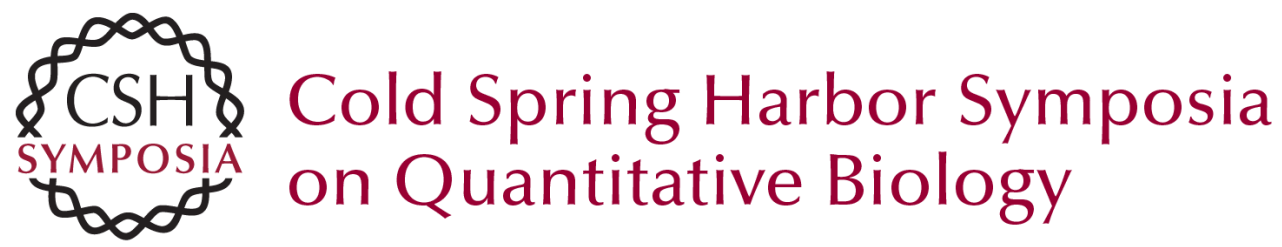

\section{Three-Dimensional Organization of Chromatids by Nuclear Envelope-Associated Structures}

Günter Blobel

Cold Spring Harb Symp Quant Biol 2010 75: 545-554 originally published online November 3, 2010 Access the most recent version at doi:10.1101/sqb.2010.75.004

References This article cites 55 articles, 25 of which can be accessed free at: http://symposium.cshlp.org/content/75/545.full.html\#ref-list-1

\section{License}

Email Alerting Receive free email alerts when new articles cite this article - sign up in the box at the Service top right corner of the article or click here. 